\title{
La innovación aplicada a la financiación de las empresas de la economía social. El caso de las inversiones de impacto
}

\author{
Nina Magomedova \\ Lluís Carreras Roig \\ Ramon Bastida-Vialcanet
}

RESUMEN: La crisis económica y financiera ha provocado una creciente desconfianza en el sistema capitalista. En los años posteriores a la crisis, surgieron diferentes iniciativas de inversores de capitales para dirigir sus inversiones a organizaciones y proyectos que produjeran un impacto social o medioambiental. Estos inversores acostumbran a priorizar la obtención de este impacto a la rentabilidad económica. En la actualidad, el mercado de las inversiones de impacto se ha desarrollado de forma importante en países como Estados Unidos, Reino Unido y Canadá. También en países de la UE como Holanda o Dinamarca. Hemos analizado el caso de la utilización de esta fórmula de financiación en el crecimiento de una empresa con impacto social, dedicada al diseño y a la comunicación. En 2016, la empresa cerró una ronda de financiación a la que acudieron diversos inversores de impacto. El análisis de esta experiencia ha puesto de manifiesto que la alineación de objetivos en torno a la generación de impacto social, fue un factor clave para el entendimiento entre ambos. Sin embargo, la falta de herramientas para medir el impacto social y la cautela de los inversores fueron factores que condicionaron el crecimiento de la empresa. En el presente artículo se explican los detalles de la financiación.

PALABRAS CLAVE: Inversiones de impacto, impacto social y medioambiental, empresa social, finanzas sociales, tercer sector.

CLAVES ECONLIT: L31, G24, 035.

Cómo citar este artículo / How to cite this article: MAGOMEDOVA, N., CARRERAS, LL. \& BASTIDA-VIALCANET, R. (2020): "La innovación aplicada a la financiación de las empresas de la economía social. El caso de las inversiones de impacto", CIRIEC-España, Revista de Economía Pública, Social y Cooperativa, 98, 127151. DOI: $107203 /$ CIRIEC-E.98.13212.

Correspondencia: Nina Magomedova, Profesora ayudante, Universitat Internacional de Catalunya, e-mail: nina.magomedova@uic.es; Lluís Carreras Roig, Profesor Titular de Escuela Universitaria, Universitat Rovira i Virgili, lluis.carreras@urv.cat (autor de contacto), y Ramon Bastida-Vialcanet, Profesor Titular, UPF Barcelona School of Management, ramon.bastida@upf.edu. 


\section{EXPANDED ABSTRACT}

\section{Innovation applied to financing of enterprises of so- cial economy. A case of impact investment}

The economic and financial crisis of 2008 caused a significant mistrust to the capitalist system. After the crisis, different initiatives of capital investors emerged to direct their investments to organizations and projects that would have a social and / or environmental impact. Among management scholars and practitioners such investments are known as impact investments.

Impact investments represent a new financing formula for projects that have a social and / or environmental impact, as well as an economic return. Investors who provide financial resources to these types of projects tend to prioritize social or environmental impact, to economic profitability. That is, they are willing to obtain a lower return in comparison with a conventional investment, as long as they can compensate for it with the achievement of a measurable social and / or environmental impact.

The impact investment market, like any other market, is a combination of capital demand to finance impact generating projects, impact capital supply, and intermediaries that help connect supply and demand. Currently, the impact investment market has developed significantly in countries such as the United States and Canada, as well as some European countries, such as the United Kingdom, The Netherlands and Denmark. These countries have been pioneers in the use of this financing formula for social projects.

Given the growing importance of the impact investment market among practitioners, as well as an increasing interest towards this phenomenon among scholars, this study is aimed to research the concept of impact investment, and puts forward a twofold objective. First, the study aims to perform a descriptive analysis of the concept and characteristics of impact investments, as well as the main existing research lines. Second, the authors present a case of the application of impact investments to finance the growth of a social enterprise.

Specifically, the authors have used the descriptive single case study method. In order to select the exemplary case for the single case study, the purposeful sampling technique is applied, which is considered especially suitable for the objectives of construction and extension of theory. Specifically, the authors have looked for a social enterprise that, on the one hand, has a clear social impact in the core of its main activity, and on the other hand, aims to economic sustainability instead of philanthropy. To choose the unique case the database of 519 social enterprises prepared by the Generalitat de Catalunya has been scrutinized. More than $50 \%$ of the social enterprises in this database are work inclusion companies, so this sector has been chosen as the most representative and important for the social and solidarity economy of Catalonia. 
In the preliminary analysis the study has excluded non-profit organizations whose main reason for including people with disabilities was purely philanthropic and not aligned with the main business. As a result, only one was chosen because of its relevant impact on the local and international community - a design studio La Casa de Carlota (LCDC).

During its five years of existence, this social enterprise has grown from a newborn start-up, to a company with a broad customer base and a subsidiary in Latin America. LCDC has reached the breakeven point in 2017, and is now in the phase of expansion. During these years, the funding strategy has been changing according to the needs of the enterprise.

Although there are certain similarities with a conventional start-up, the financing strategy has always been marked by its purpose of creating social impact. In particular, the enterprise avoided conventional investors that would look for economic profit maximization, and would turn to investors that prioritize its social impact. As the results the enterprise turned to impact investors, who did not seek short-term financial profitability, but rather prioritized creation of social impact and long-term economic sustainability. In the case of LCDC, impact investors were two non-profit foundations.

The study shows that the funding process was not an easy task for the enterprise. First, there were difficulties to measure the social impact generated. According to its founders, the impact generated by the inclusion in the work team of people with intellectual disabilities is evident, but they do not have tools to quantify this impact. In this sense, they consider that this lack of resources for measuring social impact can be an important obstacle for social enterprises to receive financing from impact investors.

Second, investment strategies of impact investors, like foundations, are characterized by being conservative and cautious. This fact had a negative impact on LCDC, because these foundations do not usually make large capital injections at once, but instead distribute them over a period of time. This fact affected LCDC's growth strategy.

There were, however, positive features in choosing impact investors as well. An interesting singularity of the impact investment was the relationship between the investor and the social enterprise. In this sense, LCDC treated its investors not only as financing providers, but as partners, linking investors in the main activity of the company, taking advantage of their experience, and treating them as team members.

Finally, the intermediary platform between LCDC and the investment foundations was of great importance so that the company could be financed through impact investors. In the case of LCDC, it was the Ship2B platform, which not only helped to connect the two agents, but also helped in the development of the action plan, the estimation of the value of the company, and the contribution of technical knowledge to the project. 
The study has some limitations. First, it only presents a single case study, which limits the generalizability of its results. The choice of this methodology was conditioned by the objectives of the study, which is to analyze a sample case of a funding strategy of a social enterprise through impact investors. However, more quantitative research should be done to contrast the results of the qualitative study. Second, the specific case of a social enterprise of work inclusion has been chosen. A cross-case comparative analysis that would include social enterprises from various industries is needed to shed more light on the nature of impact investment industry.

The study has important implications for both scholars and practitioners, because it helps to understand the nature of impact investments and sheds light on this new ecosystem. Social enterprises and impact investors occupy an important place in the economies of both developed and developing countries, and academic researchers have to pay more attention to this new type of economy.

We contribute to the existing academic research by presenting an analysis of a case of the use of this financing instrument in the growth of a company with social impact, dedicated to design and communication. In 2016, the company closed a funding round with several impact investors. The analysis of this experience has shown that the alignment of objectives around the generation of social impact, was a key factor for the understanding between the two. However, the lack of tools to measure the social impact and caution of investors were factors that conditioned the growth of the company.

KEYWORDS: Impact investment, social and environmental impact, social enterprise, social finance, third sector. 


\section{Introducción}

La crisis económica y financiera que se inició en España en el cuarto trimestre del año 2007 ha provocado una creciente desconfianza en el sistema capitalista, en el que la mayoría de las empresas tienen como principal objetivo maximizar sus beneficios para crear valor para sus accionistas. Se ha comprobado que se está produciendo un cambio en la idea de hacer negocios que tienen los representantes de la generación "millennial"(The Economist, 2017). Éstos consideran que el objetivo principal de las empresas no ha de ser únicamente maximizar beneficios, sino también mejorar la sociedad, mediante la realización de actividades que tengan un impacto social o medioambiental en su entorno.

En este sentido, Kramer y Porter (2011) ponen sobre la mesa un nuevo paradigma de creación de valor de las empresas, al que denominan valor compartido (shared value). Estos académicos consideran que las empresas no solo han de maximizar la creación de valor para sus accionistas (anterior paradigma), sino que se ha de compartir con la creación de valor medioambiental y social en la comunidad en la que están situadas. Este tipo de empresas forman una parte importante de la economía social, cuya capacidad de añadir valor social está ampliamente reconocida en el ámbito científico, social y político (Sánchez, et al., 2015). La literatura académica y el mundo profesional identifican este tipo de organizaciones con el nombre de empresas sociales y destacan su fuerte motivación de cambiar la sociedad y crear el valor social (Valcárcel Dueñas, 2012).

En este contexto surgen las inversiones de impacto. De acuerdo con el Global Impact Investing Network (GIIN), que es la red más importante a nivel internacional que agrupa a los actores más relevantes del ecosistema de las inversiones de impacto, éstas se definen como las "inversiones realizadas en empresas, organizaciones y fondos con la intención de generar impacto social y medioambiental, además de rentabilidad financiera. Estas inversiones pueden realizarse tanto en mercados emergentes como desarrollados y buscar rentabilidades inferiores al mercado o de mercado, dependiendo de las circunstancias" (GIIN, 2018).

Las inversiones de impacto tienen dos características específicas. Son inversiones dirigidas a empresas o entidades con una clara misión social o medioambiental. Por lo tanto, realizan actividades destinadas a producir productos $u$ ofrecer servicios que cubren necesidades sociales o medioambientales. Y son inversiones que tienen un doble resultado. Tienen un retorno económico sobre el capital invertido y tienen un impacto social o medioambiental medible. De este modo, los inversores de impacto cumplen con el objetivo de invertir sus capitales en empresas sostenibles que realicen actividades con impacto social y, al mismo tiempo, obtienen un beneficio económico por el capital invertido (Bastida, 2018). 
Este tipo de inversiones representan una innovación importante en el modelo de financiación de las empresas y entidades que desarrollan su actividad en el sector social, y que históricamente se habían financiado con dinero público. Para estas empresas suponen una oportunidad de cambiar el modelo de financiación para mejorar su autonomía financiera y asegurar su sostenibilidad en el tiempo. Las inversiones de impacto también son una nueva fuente de financiación para emprendedores sociales que quieran poner en marcha una start-up social. En este sentido, en ciudades como Barcelona, se está empezando a crear un ecosistema de creación y desarrollo de empresas sociales.

Diversos autores consideran que las inversiones de impacto van más allá de las inversiones socialmente responsables. Se les atribuye a las primeras una mayor proactividad para resolver problemas sociales o medioambientales (Höchstädter \& Scheck, 2015). Desde el punto de vista de la investigación, las inversiones de impacto constituyen un incipiente campo de investigación que tiene un considerable interés y potencial de crecimiento, pero que aún está en la fase inicial de su desarroIlo. Asimismo, las inversiones de impacto social constituyen un área de investigación y práctica y un paradigma académico propio (Daggers \& Nicholls, 2016).

Este trabajo tiene un doble objetivo. En primer lugar, realizar un análisis descriptivo del concepto y las características de las inversiones de impacto, así como de las principales líneas de investigación existentes. En segundo lugar, analizar un caso práctico real de aplicación de las inversiones de impacto para financiar el crecimiento de una empresa social.

En el siguiente apartado, se realiza una presentación conceptual de los aspectos clave de las inversiones de impacto. En el tercer apartado, se realiza un análisis de la revisión de la literatura sobre inversiones de impacto realizada por Daggers y Nicholls (2016). En los apartados cuarto y quinto, se analiza la metodología y los resultados, respectivamente, del caso práctico de estudio. En el apartado sexto, se realiza una discusión sobre los resultados obtenidos. Finalmente, en el apartado séptimo se presentan las conclusiones.

\section{Las inversiones de impacto}

El concepto de las inversiones de impacto ha surgido como una respuesta a las necesidades de las empresas sociales, la clave de las cuales se centra en la creación de valor social o medioambiental más allá del rendimiento económico (Priede, et al., 2014). A continuación, realizamos una presentación teórica o conceptual de los aspectos relevantes de las inversiones de impacto. 


\subsection{Concepto y características}

Además de la definición de las inversiones de impacto ofrecida por el GIIN, existen otras definiciones del término. El informe "Impact Investing in Europe. Extract from European SRI Study 2014" (Eurosif, 2014), incluye una tabla resumen de las definiciones y de las características principales de las inversiones de impacto, elaboradas por las instituciones y organismos internacionales más relevantes relacionados con este tipo de inversiones. La Organización para la Cooperación y el Desarrollo Económico (OCDE) define las inversiones de impacto como "las aportaciones de financiación a las organizaciones con una expectativa explícita de un retorno, a la vez, social y financiero" (OECD, 2018). Como indica esta definición, una de las características clave de las inversiones de impacto es la búsqueda conjunta del impacto social de las inversiones y de una rentabilidad financiera. Esta condición implica que la rentabilidad financiera de las empresas financiadas no podrá disociarse de los objetivos de impacto.

Por su parte, el Fondo Monetario Internacional (FMI) define las inversiones de impacto como "un tipo de inversión que intencionadamente busca crear una rentabilidad financiera y un impacto social o medioambiental positivo". Y añade que "busca intencionadamente conseguir este doble objetivo social/medioambiental y financiero. Teniendo en cuenta que la rentabilidad financiera podrá estar a nivel de mercado, por encima del mercado, o por debajo del mercado" (Eurosif, 2014).

En las definiciones anteriores podemos observar que hay varias características que se repiten en todas ellas y que las caracterizan frente a otros tipos de inversiones:

a) Búsqueda conjunta del impacto social o medioambiental y de la rentabilidad financiera.

b) Impacto social o medioambiental intencionado.

c) Impacto social o medioambiental medible.

d) Rango de expectativas de rentabilidad financiera.

Podemos afirmar que la medición y el rendimiento de cuentas del impacto social o medioambiental es el principal factor diferencial de las inversiones de impacto respecto a las inversiones tradicionales.

\subsection{Ecosistema de las inversiones de impacto}

El mercado de las inversiones de impacto, como cualquier otro mercado, es una combinación de demanda de capital para financiar organizaciones generadoras de impacto, de oferta de capital de impacto y de intermediarios que ayudan a conectar la oferta y la demanda. En este sentido, hablamos de los inversores de impacto, los intermediarios, los generadores de impacto y los compradores de impacto (Bastida, 2018). 
- Los inversores de impacto son los actores encargados de aportar el capital necesario para financiar las entidades y empresas generadoras de impacto.

- Los intermediarios sirven para conectar los inversores con las organizaciones generadoras de impacto, en aquellos casos en los que los inversores no invierten directamente en estas organizaciones. Este tipo de actores incluyen entidades de banca ética y tradicional, gestores de fondos de inversión y plataformas de financiación, entre otros (Mendell \& Barbosa, 2013; Nicholls \& Emerson, 2015a, 2015b; Rajan et al., 2014).

- Los generadores de impacto son diferentes tipos de organizaciones que tienen una misión social, definen objetivos y miden su realización (Lyons and Kickul, 2013). Son las organizaciones receptoras de las inversiones de impacto. Pueden ser tanto organizaciones del sector social (fundaciones, cooperativas sociales, etc.) como empresas tradicionales con impacto. No existe un consenso a la hora de identificar qué tipo de organizaciones son susceptibles de ser receptoras de las inversiones de impacto (McWade, 2012; Mulgan, 2015; Nicholls, 2010).

- Los compradores de impacto son entidades y personas que aportan los ingresos necesarios para las empresas que generan impacto. Los compradores de impacto pueden incluir tanto a administraciones públicas como a consumidores, empresas o fundaciones (Nicholls \& Schwarz, 2014; Ormiston \& Seymour, 2014).

\subsection{Instrumentos financieros}

Las inversiones de impacto se materializan a través de un conjunto heterogéneo de instrumentos que sirven para captar fondos en los mercados financieros destinados a financiar inversiones que permitan obtener, a la vez, una rentabilidad financiera y social o medioambiental. Entre los instrumentos de captación de fondos destacan el capital riesgo de impacto social, los bonos verdes y los bonos de impacto social, y las plataformas de financiación participativa de proyectos con impacto social.

El capital riesgo de impacto pretende obtener una rentabilidad financiera para el inversor, además de impulsar soluciones de mercado a cuestiones sociales y medioambientales. Es una inversión a largo plazo con un apalancamiento elevado y escasa liquidez. Este tipo de instrumentos sirven para financiar inversiones importantes, pero no resuelven el problema de la generación de ingresos, que es uno de los grandes retos de las entidades y empresas financiadas con las inversiones de impacto. Algunos fondos de capital riesgo contemplan la posibilidad de ofrecer asistencia técnica a las entidades y empresas en las que invierten, especialmente si éstas se encuentran en una fase inicial.

Los bonos verdes tienen las mismas características financieras de los bonos convencionales, pero están diseñados para financiar proyectos e iniciativas que pretenden conseguir beneficios medioambientales. Los bonos verdes se están consolidando en los mercados financieros internacionales como una de las mejores alternativas a la hora de invertir en productos financieros relacionados 
con el cambio climático. En 2017, el Ayuntamiento de Barcelona realizó la primera emisión de bonos verdes y bonos sociales con un capital total de $35 \mathrm{M}$ de euros (Bastida, 2018).

Los bonos de impacto social permiten a los inversores financiar intervenciones que persiguen mejorar la situación de determinadas cuestiones sociales de especial interés para la Administración Pública (Jackson, 2013). Se basan en un contrato entre un inversor, una administración pública, y una entidad o empresa social (Joy \& Schields, 2013; McHugh et al., 2013). El inversor obtiene un retorno financiero si como resultado de la intervención se obtienen los resultados previstos (pago por resultados). Este tipo de bonos son un ejemplo de colaboración público-privada. Se crearon en el año 2010 en el Reino Unido, pero actualmente se pueden encontrar en países como EEUU, Alemania, Bélgica u Holanda (Eurosif, 2014).

Las plataformas de financiación participativa son una modalidad de financiación a través de la cual se reúnen aportaciones de fondos procedentes de un conjunto de inversores, profesionales y minoristas, a través de plataformas online, con el objetivo de financiar un proyecto. Hay varios tipos de financiación participativa, pero para que puedan ser considerados inversiones de impacto, los inversores deben buscar un retorno financiero y los proyectos deben buscar un impacto positivo y medible en el ámbito social o medioambiental. Recientemente, se ha creado un nuevo tipo de financiación participativa llamado "crowdimpacting" dirigido a empresas que combinen un buen modelo de negocio con potencial de crecimiento y que produzcan un impacto positivo en la sociedad y en el medio ambiente (Mendell \& Barbosa, 2013).

\section{Revisión de la literatura}

El análisis de la literatura sobre inversiones de impacto realizado por Daggers y Nicholls (2016), indica que existe un número muy superior de artículos e informes profesionales en comparación con artículos académicos. En este sentido, se confirma que la investigación en este campo se encuentra en una fase inicial, pero está experimentando un crecimiento muy destacable en los últimos años.

Existen organizaciones situadas principalmente en el Reino Unido, Estados Unidos y Canadá, que son muy activas en el patrocinio, publicación o producción de informes profesionales con el fin de promover las inversiones de impacto mediante el apoyo de los gobiernos de estos países. Este hecho puede explicar la diferencia entre la producción profesional y la académica. 
Los artículos académicos se han centrado en aspectos teóricos y conceptuales o descriptivos 0 de mapeo, realizados mediante argumentos teóricos 0 datos secundarios obtenidos a partir de información sectorial o del análisis de la literatura. De los 73 artículos académicos seleccionados por Daggers y Nicholls, 20 artículos (27\%) no incluyen ningún tipo de datos, se centran en presentar argumentos teóricos sobre aspectos relacionados con las inversiones de impacto. 34 artículos (47\%) utilizan datos secundarios basados en cifras generales disponibles en la literatura, o en información más estructurada obtenida a partir de un análisis de la misma. Los artículos empíricos son muy escasos y se basan en datos primarios obtenidos a partir de entrevistas a los inversores de impacto. Solo 19 artículos (26\%) se basan en datos primarios obtenidos a partir de encuestas, principalmente, a inversores de impacto.

Del análisis de la literatura, se desprende la necesidad de incrementar los estudios empíricos para contribuir al estudio de la aplicación práctica de las inversiones de impacto. En este sentido, en los próximos apartados se analiza el uso de las inversiones de impacto para financiar el crecimiento de una empresa social.

\section{Metodología}

Dada, por un lado, la naturaleza exploratoria de la pregunta de investigación y, por otro lado, las escasas evidencias empíricas de inversiones de impacto en la literatura académica, la aplicación de la metodología de estudio de caso holístico se considera adecuada, debido a su capacidad de construcción teórica (Yin, 2017). Específicamente, hemos utilizado el método de estudio de caso único descriptivo siguiendo los pasos propuestos por Eisenhardt (Eisenhardt, 1989), quien sugiere empezar con la definición de la pregunta de investigación y proceder con la selección de casos. Para seleccionar el caso ejemplar para el estudio de caso único, se aplica la técnica del muestreo intencional ("purposeful sampling") (Patton, 2005), que se considera especialmente adecuada para los objetivos de construcción y ampliación de teoría (Eisenhardt \& Graebner, 2007). En concreto, buscamos una empresa social según la definen Höchstädter y Scheck (2015): "Una empresa que tiene un claro impacto social alineado con su actividad principal y fines de sostenibilidad económica y no de filantropía". Para elegir el caso único hemos utilizado la base de datos de las empresas sociales elaborada por la Generalitat de Catalunya. La base incluye 519 empresas sociales, de las cuales más de $50 \%$ son empresas de inserción, por lo que hemos elegido este sector como el más representativo e importante para la economía social y solidaria de Cataluña. 
En el análisis preliminar hemos rechazado las asociaciones sin ánimo de lucro y las empresas cuyo motivo principal de incluir las personas con discapacidades fue puramente filantrópico y no alineado con el negocio principal, ya que el objetivo fue elegir una empresa con actividad económica, con un potencial de generar valor económico y, además, crear el impacto social a través de la inserción de las personas en riesgo de exclusión social como, por ejemplo, personas con discapacidad intelectual. Como resultado, solo una fue elegida debido a su impacto relevante en la comunidad local e internacional - el estudio de diseño La Casa de Carlota (LCDC). LCDC es una empresa donde además de diseñadores profesionales y estudiantes de las escuelas de arte, trabajan personas con discapacidad intelectual. La principal diferencia de LCDC radica en el hecho de que esta empresa contrata a personas con discapacidades por su talento creativo y la visión del mundo singular.

\subsection{Recogida de datos}

Los datos se recopilaron a través de entrevistas semiestructuradas en profundidad, con dos cofundadores de la empresa, en dos rondas, a través de conversaciones telefónicas y cara a cara grabadas y transcritas rigurosamente (ver tabla 1 y tabla 2). A continuación, las transcripciones de las entrevistas fueron contrastadas con las notas de campo hechas durante las mismas. Después de haber ordenado y analizado los datos recopilados durante las entrevistas y trianguladas con la información en internet y prensa, los resultados preliminares fueron enviados a los entrevistados para determinar si se omitieron las ideas clave y para obtener observaciones adicionales del caso.

\section{Tabla 1. Descripción de la muestra}

\begin{tabular}{|l|l|l|}
\hline & Entrevistado 1 & Entrevistado 2 \\
\hline Nombre & José María Batalla & Sergi Capell \\
\hline Puesto & $\begin{array}{l}\text { Fundador, } \\
\text { Director Creativo }\end{array}$ & $\begin{array}{l}\text { Fundador, } \\
\text { Director General }\end{array}$ \\
\hline $\begin{array}{l}\text { Fecha y duración de la primera ronda de } \\
\text { entrevistas }\end{array}$ & $\begin{array}{l}26 \text { de abril, 2018 } \\
\text { cerca 60 minutos }\end{array}$ & $\begin{array}{l}30 \text { de mayo, 2018 } \\
\text { cerca 30 minutos }\end{array}$ \\
\hline $\begin{array}{l}\text { Fecha y duración de la segunda ronda de } \\
\text { entrevistas }\end{array}$ & $\begin{array}{l}9 \text { de mayo, 2018 } \\
\text { cerca 30 minutos }\end{array}$ & $\begin{array}{l}13 \text { de junio, 2018 } \\
\text { cerca } 90 \text { minutos }\end{array}$ \\
\hline
\end{tabular}




\section{Tabla 2. Guión de entrevistas}

$1 \quad$ ¿Cómo comenzó el negocio?

¿Cuál es el origen de los fundadores y la experiencia previa? ¿Cómo influyen las características personales y los valores en la creación de un modelo de negocio como LCDC?

2 ¿Cómo elaboró el plan de negocios e inversión para LCDC?

¿Qué diferencias existen en la preparación de un plan de inversión para un estudio de diseño "convencional" en comparación con el de LCDC? ¿Hay algún coste adicional relacionado con personas con discapacidades? ¿Qué importancia tiene el factor de apoyo gubernamental relacionado con la inclusión laboral? ¿Cómo se definió el impacto social en el plan de negocios inicial? ¿Estaba incluido en el balance previsional? ¿Cómo comenzó la empresa a buscar financiación? ¿Cuál fue la reacción de los inversores tradicionales a su modelo de negocio? ¿Cuándo surgió la idea de atraer inversores de impacto? ¿Qué diferencias existen en el trato con inversores de impacto en comparación con inversores tradicionales?

$3 \quad$ ¿Cómo define y mide el impacto social creado por su empresa?

¿Cómo mide la empresa sus resultados? ¿Qué componentes son más/menos importantes? ¿Cómo se evalúa/compara el resultado con los de los competidores? ¿Cuál es el rol de los certificados (como B Corp) en la medición de los resultados?

$4 \quad$ ¿Cómo podría describir la estructura financiera de LCDC hoy en día?

¿Qué tipo de financiación utilizan? ¿Quién son los socios/inversores? ¿Cuáles son las relaciones entre LCDC y los socios? ¿Cómo han elegido a los socios? ¿Qué tipo de retribución tiene el capital? ¿Cuándo ha llegado LCDC al punto de equilibrio?

\subsection{Explicación del caso}

LCDC es un estudio de diseño profesional fundado por José María Batalla y Sergi Capell en 2013. Sus servicios principales son las campañas de promoción, publicidad y embalaje. Desde su creación ha desarrollado proyectos para importantes empresas privadas nacionales y multinacionales, y para entidades del sector público. La ventaja competitiva de esta empresa se basa en disponer de un equipo creativo que se sostiene en base a tres pilares fundamentales (figura 1). 


\section{Figura 1. Tres pilares de LCDC}

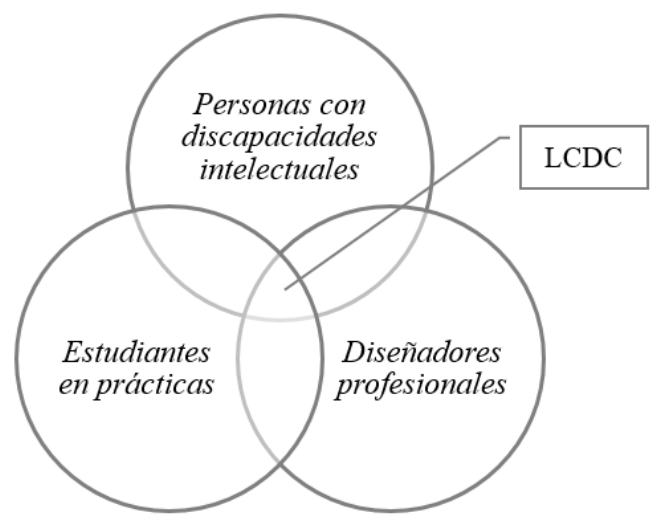

El primer pilar son personas con discapacidades intelectuales, como síndrome de Down o autismo y con un gran talento artístico; diseñadores con una manera singular de percibir el mundo, lo cual produce como resultado la creación de obras únicas de arte y diseño. El segundo pilar son los estudiantes de las escuelas de arte y diseño. Son personas con una visión fresca e ingeniosa, sin miedo a proponer algo distinto. LCDC les da la oportunidad de romper con las barreras que algunos se imponen durante sus estudios. Y, por último, el tercer pilar del equipo de diseño son diseñadores profesionales, capaces de sintetizar ideas. Algunos de ellos son de otros países y también, como parte de la política de empresa, algunos proceden del programa de prácticas profesionales para estudiantes que desarrolla el estudio.

La empresa existe desde hace cinco años y está en plena fase de expansión. La estrategia de captación de fondos ha ido cambiando según las necesidades surgidas en las diferentes etapas de su existencia. En el siguiente apartado exponemos la diversidad de estrategias de búsqueda de financiación y los tipos de inversiones que la empresa empleó y obtuvo, respectivamente. 


\section{Resultados}

En los 5 años de su existencia LCDC ha pasado de la fase start-up a ser ya una empresa consolidada; ha logrado el punto de equilibrio y ahora está en plena expansión. A primera vista, su experiencia de búsqueda de fondos económicos puede parecer semejante a una start-up habitual. Sin embargo, el hecho de ser una empresa social ha influido considerablemente en su trayectoria. Este apartado presenta la descripción y análisis de la experiencia de búsqueda de financiación de LCDC.

\subsection{Necesidades de empresa}

Según la experiencia de LCDC, uno de los factores clave que influyeron en la selección de los inversores fue el ciclo de vida de la empresa. A la hora de lanzar la empresa, los fundadores utilizaron sus propios fondos para satisfacer las necesidades de la empresa en su etapa inicial. Debido a las características específicas del sector, la empresa no necesitaba grandes inversiones de entrada, y las pequeñas inyecciones financieras fueron suficientes. Durante los primeros cinco años, esta estrategia fue suficiente para la supervivencia de la empresa, para adquirir experiencia y captar los primeros clientes. Sin embargo, recientemente, LCDC entró en la etapa de plena expansión. En 2015-2016 resultó obvio que, para mantener el crecimiento, LCDC necesitaba una estrategia de captación de inversiones más intensiva. En concreto, para aumentar el equipo profesional y disponer de las instalaciones adecuadas para el tamaño de la empresa, los fundadores valoraron las necesidades económicas en 150 mil euros, y empezaron a buscar inversores. A la hora de conseguir los inversores, un factor al que tuvieron que enfrentarse fue el hecho de que los inversores no les entregaron el capital de una sola vez, sino que lo distribuyeron a lo largo de un año. Según la experiencia de LCDC, esta estrategia no ayuda, sino que perjudica el desarrollo previsto en el plan de expansión inicial. Según Sergi Capell:

"Hicimos la salida de 150.000 euros de inversiones, y resultó que nos la dieron durante un año. Y eso mata, porque te planteas una estrategia en base del crecimiento que captarás, y resulta que no puedes crecer, como lo has planteado. Recibir todo el capital en un único pago hubiera sido lo idóneo".

La estrategia de distribuir la inversión a lo largo de un periodo de tiempo largo no suele caracterizar las inversiones tradicionales. En el caso de LCDC, los inversores son fundaciones privadas, que se financian con donaciones. La política de realizar sus inversiones en pequeñas inyecciones 
de capital a lo largo de un período de tiempo, puede estar relacionada con el carácter conservador y cauteloso de este tipo de organizaciones. Además, la experiencia en gestión capital riesgo de las fundaciones es limitada, y puede afectar negativamente a la hora de otorgar fondos a las empresas jóvenes, por su aversión a tomar decisiones arriesgadas.

\subsection{Selección de financiación}

La elección de los inversores de impacto estuvo influida por el hecho de ser una empresa social. Primero, los fundadores acudieron a sus propios fondos para lanzar LCDC. Como hemos mencionado anteriormente, los fondos propios fueron suficientes para mantener los primeros años de la vida de la empresa. Sin embargo, en la etapa de expansión, los fundadores decidieron que los riesgos podrían ser demasiado elevados, y empezaron a buscar financiación externa. El segundo recurso podría haber sido acudir a la banca tradicional. Por la naturaleza del sistema bancario español, los créditos a las empresas start-up están muy limitados y suelen consistir en importes de pequeña cuantía, de forma que para acceder a créditos de importes más elevados, el banco requiere el aval de los fundadores. La propuesta de la banca tradicional no pareció adecuada a los fundadores para afrontar la etapa de expansión, puesto que ésta requería importantes inyecciones de capital. Por esa razón acudieron a los inversores de capital riesgo.

La captación de capital riesgo fue una muy buena experiencia para LCDC. Por un lado, los inversores de capital riesgo estaban buscando inversiones con baja inversión inicial y alto rendimiento a corto plazo. Esta situación se intensificó en los últimos años con el boom de las empresas tecnológicas y de aplicaciones, cuyo retorno económico es muy rápido. Por otro lado, los inversores de capital riesgo no están acostumbrados a las empresas sociales; no conocen el sector de la economía social y este desconocimiento provoca confusión en la comprensión de los objetivos de las empresas como LCDC. Según Sergi Capell:

"Las empresas sociales las ven como unos chiringuitos. No se entiende muy bien qué es esto -una ONG, una fundación o una asociación-. No se sabe qué es una empresa con ánimo de lucro y con impacto social. De ahí la falta de confianza y contradicciones en los objetivos finales".

La alternativa era buscar los inversores de impacto, los cuales tienen como objetivo obtener no solo rentabilidad económica, sino también impacto social. Este tipo de inversores han aparecido en España hace pocos años, y el sector está en las etapas incipientes. La mayoría de los inversores de impacto son fundaciones, nacionales e internacionales, que se financian mediante donaciones. En España, su experiencia como inversores de impacto es todavía muy escasa, y esto plantea ciertas limitaciones a las empresas sociales que reciben sus inversiones. Sin embargo, LCDC apostó por este tipo de inversor debido a una serie de factores, que se detallan a continuación. 


\subsection{Factores clave en la selección de inversiones de impacto}

Entre los factores principales que influyeron en la decisión de financiarse mediante los inversores de impacto, se pueden distinguir: la estabilidad en la financiación y orientación a largo plazo, el requisito de generar un impacto social bien definido, la alineación de los objetivos y valores entre la empresa y el inversor, y la vinculación del inversor al negocio de la empresa social.

En primer lugar, los inversores de impacto dan tranquilidad a las empresas sociales. En el caso de las empresas que realizan actividades de inclusión laboral de personas con discapacidades esta tranquilidad financiera es crucial. El ambiente laboral de este tipo de empresas es muy frágil, ya que la presión en el sector del diseño es elevada, debido al carácter fraccionado del trabajo por proyectos y a la alta necesidad de adaptación de la estructura organizativa. Eso significa que, para su funcionamiento fluido, y para alcanzar su objetivo principal de generar impacto social, la empresa necesita un margen de tiempo para devolver la financiación obtenida. En este sentido, los inversores de LCDC le dan a la empresa la necesaria tranquilidad y paciencia, incluso hasta el punto que la misma empresa reconoce que los inversores deberían ser más exigentes. Sin embargo, esta estrategia ha ayudado a LCDC a lograr los resultados de alto impacto social y rendimiento económico.

En segundo lugar, los inversores de impacto priorizan la generación de impacto social sobre la rentabilidad económica. Este fue el caso de los inversores de LCDC. Según José María Batalla:

"Nuestros inversores buscaban no tanto el "payback", sino el impacto, buscaban una inversión a largo plazo."

En otras palabras, la rentabilidad financiera de la empresa es menos importante que el impacto social que esta empresa debería generar. Por tanto, se supone que para medir la rentabilidad global (financiera y social) de la empresa, se debería medir el impacto social, para ver cuál es retorno social de inversión ("social return on investment"). Cuando preguntamos si los inversores piden información sobre el impacto generado por la empresa y las posibles métricas, resulta que LCDC no está obligada a proporcionar esta información a sus inversores. Según Sergi Capell:

"Nuestros inversores no piden esta información, pero no significa que no tienes que tenerla. Tienes que exigirla tú mismo, porque los inversores no saben que quieren, ni que necesitan para evaluar tu proyecto de negocio. Has de demonstrar el impacto que generas, pero también la rentabilidad. Tienes que saber qué impacto generas tanto social como económico."

La empresa enfatiza que precisamente la capacidad de demostrar el impacto social le ha ayudado a ganar la confianza de sus inversores, porque les diferenciaba de otras agencias de diseño y publicidad. Según Sergi Capell: 
"Una de las cosas más importantes en la búsqueda de inversores es demostrar el valor añadido. En nuestro caso es la capacidad de meter dentro del equipo gente que piense de manera diferente. La manera de parir la creatividad en nuestra agencia es muy distinta, y eso es lo que forma nuestro valor diferenciado. En términos de inversores siempre hemos salido con este mensaje."

En tercer lugar, LCDC eligió a los inversores de impacto porque sus valores y objetivos están alineados con los de la empresa. Según José María Batalla:

Nuestra reflexión fue que quizás sería más fácil buscar la financiación de los bancos, pero llegamos a la conclusión que nos gustaría que nos acompañaran inversores con ideas alineadas a las nuestras.

Por el mismo motivo no acudieron a la banca ética, que mayoritariamente sigue la estrategia de inversiones socialmente responsables, excluyendo a ciertas empresas, y no la estrategia de creación del impacto social positivo (Höchstädter \& Scheck, 2015). Las fundaciones que apoyaron LCDC, una de la cuales es un centro de trabajo especial para las personas con discapacidades, tienen participaciones en otras empresas sociales, y sus actividades están vinculadas con las actividades de LCDC. Esto les ayuda a entender las necesidades de la empresa en las fases más complicadas, como la de la expansión.

Por último, la alineación de los valores y objetivos de la empresa social con los de su inversor de impacto, crea una fuerte vinculación entre ellos, convirtiéndolos en socios. Compartiendo su experiencia de captación de financiación, los fundadores destacaron la existencia de dos tipos de inversores. El primer tipo son "los traders de bolsa" - los inversores, que proporcionan la financiación sin vinculación activa en el negocio mismo y con el único objetivo de maximizar la rentabilidad de sus inversiones. Este tipo de inversores tiene una visión a corto plazo, buscan el retorno rápido, sin interesarse en la creación del impacto social. El segundo tipo son los "inversores-socios", que tienen la visión a largo plazo y están interesados en el éxito de la empresa, tanto a nivel económico como en el aspecto del impacto social. Tener este tipo de inversores-socios puede ser vital para un emprendedor social. Según Sergi Capell:

"Son este ejemplo de inversores a quien dices: yo te necesito, no solo por el dinero, sino por lo que tú sabes, y quiero que tú te vincules. Yo no he hecho la ronda de inversión, lo que he hecho es una ronda de recursos humanos, lo que he hecho me ha convertido en un headhunter." 


\subsection{Instrumentos de financiación}

Durante el proceso de preparación de la ampliación de capital, LCDC acudió a la ayuda de una plataforma, la fundación Ship2B, situada en Barcelona, y dedicada a la aceleración de proyectos empresariales de alto impacto social, y que cuenta con una importante red de inversores de impacto. Esta fundación ayudó a elaborar el diseño del plan de negocio, que incluía las proyecciones financieras de la empresa para los siguientes años. En base a esta información, la empresa hizo su propia valoración, y presentó este plan a las dos fundaciones - inversores de impacto. Estos inversores entraron con una participación en el capital social de la empresa, y ahora disponen de los mismos derechos y obligaciones que cualquier accionista, aunque los fundadores mantienen la mayoría de las acciones y el control de la gestión de la empresa. Además de inyectar capital, estas fundaciones también aportan conocimientos técnicos al proyecto.

La etapa de valoración de proyecto fue clave, porque permitió a los fundadores obtener la financiación necesaria y mantener el control de la empresa:

"Es importante establecer el precio que te permita acudir a la inversión necesaria sin perder el control sobre la empresa. Para nosotros fue vital, porque es el proyecto de nuestra vida, y no puedes entrar y salir al negocio."

Este motivo explica también la búsqueda de inversores con la visión a largo plazo, que se vinculan a la empresa y contribuyen con su conocimiento a la gestión de la misma. Los motivos de la creación de LCDC son profundamente personales; la empresa representa los valores fundamentales de sus creadores, y la alineación de los valores e intereses de los inversores es crucial para el buen funcionamiento de LCDC a largo plazo.

\section{Discusión}

El objetivo principal de este estudio es analizar un caso real de aplicación de las inversiones de impacto para financiar el crecimiento de una empresa social. Para lograr este objetivo hemos elegido un estudio de diseño, cuyo equipo creativo incluye personas con discapacidad intelectual. La empresa tiene fines lucrativos y su cartera de clientes incluye tanto empresas comerciales interesadas puramente en la calidad del diseño elaborado por el equipo, como las entidades públicas y no lucrativas que quieren apoyar la empresa por su impacto social -inserción laboral de las personas con discapacidad intelectual-. En este apartado presentamos una discusión crítica de los resultados obtenidos. 
Como hemos comentado en el apartado del marco teórico, la característica principal de los inversores de impacto que les diferencia frente a otros tipos de inversores, es la búsqueda de un impacto social y/o medioambiental intencionado y medible (Bastida, 2018; Eurosif, 2014; OECD, 2018; Nicholls, 2010). El caso analizado ha revelado que los inversores de LCDC sí buscan el impacto tanto de forma intencional como medible, pero en realidad la empresa se enfrenta con ciertas dificultades para demostrar este impacto. Por un lado, los entrevistados de LCDC han manifestado su preocupación con respecto a la dificultad de demostrar su impacto medible, y las dificultades relacionadas con este hecho en la búsqueda de financiación.

Por otro lado, y según los fundadores de LCDC, los inversores mismos no piden indicadores de impacto concretos debido a que son conscientes que la empresa no dispone de esta información, lo cual pone de manifiesto una cierta contradicción entre las motivaciones iniciales y las prácticas reales. Los fundadores subrayan la necesidad urgente de tener las herramientas que permitan medir el impacto generado por las empresas sociales, para crear mayor transparencia y confianza entre empresas e inversores. En su ausencia, los inversores de impacto confían en la percepción cualitativa del impacto creado por la empresa social, que en ciertos casos puede ser perjudicial para ambas partes y crear barreras que dificultan su colaboración (Brandstetter \& Lehner, 2015; Diouf, 2015; Seddon et al., 2013). En este sentido, tanto la investigación académica, como los profesionales del sector de la economía social señalan la importancia de crear herramientas universales que permitan cuantificar el impacto social y ayuden a los inversores de impacto y las empresas sociales a la hora de evaluar las actividades de estas últimas y tomar decisiones de inversión.

En cuanto al ecosistema de las inversiones de impacto, el marco teórico destaca los siguientes actores: inversores, intermediarios, generadores del impacto y compradores del impacto (Bastida, 2018). En este sentido, el caso analizado es un ejemplo en el que se pueden detectar los 4 actores. En primer lugar, los inversores de impacto son las fundaciones cuyas actividades clave están alineadas con el impacto generado por LCDC - son fundaciones de inserción laboral y social de las personas con discapacidad. Aunque las fundaciones no suelen actuar como inversores profesionales, en el caso de LCDC la fundación-inversora veía la alineación completa de sus objetivos con los de la empresa, lo cual le dio la confianza necesaria para invertir el dinero de sus donantes en LCDC. En segundo lugar, para poder acceder a esta fundación, la empresa necesitaba un intermediario para dar visibilidad no solo al producto final generado por empresa, sino también al impacto social generado en el proceso de la elaboración del producto. En el caso de LCDC, este intermediario fue la plataforma de inversión Ship2B. Esta plataforma tiene como objetivo poner en contacto empresas que generen impacto social y/o medioambiental con inversores que quieran invertir en este tipo de empresas, creando así un ecosistema centrado en el impacto. En tercer lugar, la empresa-generadora del impacto, es la misma empresa social, que genera un producto final (diseño), creando un impacto social (dando trabajo a las personas con discapacidad intelectual). El hecho de crear puestos de trabajo de alta calidad y calificación (diseñadores gráficos), pagándoles los sueldos ajustados a la propuesta salarial del sector, crea un impacto todavía más importante, ya que cambia el paradigma social, donde las personas con 
discapacidad están tratadas solamente por sus discapacidades y no por sus habilidades. En este sentido, el impacto creado por LCDC es doble: primero, consigue la inserción laboral de las personas con discapacidad y, segundo, cambia el paradigma social hacia estas personas. Y, en cuarto lugar, los compradores de impacto son los clientes-empresas tanto privadas como públicas, que compran los diseños de LCDC. En algunos casos, los clientes priorizan el impacto creado por LCDC a la hora de tomar la decisión de colaborar con esta empresa. Éste sería el caso de las entidades públicas (ayuntamientos, fundaciones públicas, etc.). En cambio, en otros casos, las empresas están más interesadas en el producto final de alta calidad generada por LCDC. De forma que en estos casos, estas empresas-clientes no están tan interesadas en el impacto social de LCDC, como en el mismo diseño, único y no convencional. El hecho de tener una cartera de clientes tan amplia diferencia LCDC de entidades cuyos fines son puramente caritativos y solidarios, y la convierte en una empresa económicamente sostenible cuya actividad principal genera un impacto social importante, alineándola con la definición de empresa social, aceptada por OCDE (OECD, 2018).

Esta compleja estructura del sector de las inversiones de impacto, se encuentra todavía en una fase muy incipiente, y requerirá de más tiempo para lograr un amplio desarrollo. Sin embargo, estos avances demuestran la importancia de este sector para el futuro de la economía y la investigación académica (Bell \& Haugh, 2015).

En cuanto a los instrumentos de captación, en la literatura sobre las inversiones de impacto se destacan los siguientes: los fondos de capital riesgo social, los bonos verdes, los bonos sociales y las plataformas de financiación participativa de proyectos con impacto social; en cuanto a los instrumentos de financiación, se señalan la deuda, el capital social o una mezcla de los dos.

A lo largo de la vida de LCDC, la empresa optó en la fase inicial por el capital propio y, en la fase de crecimiento, por los inversores de impacto. La elección del capital propio está basada, por una parte, en la necesidad de mantener la autonomía en la toma de decisiones y, por otra parte, en la falta de atractivo de la actividad de LCDC para los inversores del sector convencional (banca o capital riesgo). En cuanto a las inversiones de impacto, el motivo principal para elegir este tipo de inversor fue la alineación de motivaciones y prioridades entre LCDC y los inversores, centradas en la prevalencia del impacto por encima de generación de retornos económicos. Esta fuente de financiación podría parecer óptima para las empresas sociales, ya que los inversores de impacto no suelen poner presión en términos de retornos económicos, ni suelen imponer un control excesivo sobre las actividades de este tipo de empresas. En este sentido, a diferencia de las empresas convencionales, las empresas sociales pueden necesitar un mayor margen de tiempo y expectativas más bajas a nivel económico en las primeras etapas de su ciclo de vida, y los inversores de impacto lo tienen en cuenta y lo permiten.

Sin embargo, en el análisis del caso de LCDC hemos detectado ciertas desventajas de este tipo de inversores. Como hemos visto en el apartado anterior, la empresa ha logrado obtener la financiación necesaria, pero la forma de proporcionar los recursos económicos fue poco convencional 
-las fundaciones preferían dividir el total de la inversión y hacer pequeñas aportaciones a lo largo de varios años-. Este planteamiento de financiación es algo parecido al sector público (Nicholls et al., 2015), y puede reducir la eficacia para las empresas que operan en sectores altamente competitivos (Seddon et al., 2013). La razón de este comportamiento puede ser debida a que las fundaciones que dependen de las donaciones suelen ser excesivamente precavidas en sus decisiones de inversión, sobre todo por la falta de experiencia, ya que no son inversores profesionales. Sin embargo, su interés por las empresas sociales significa que consideran a este tipo de entidades como una herramienta muy efectiva para lograr sus objetivos y, por lo tanto, la colaboración entre este tipo de entidades podría crecer en el futuro. En este sentido, para que ambas partes puedan maximizar el beneficio de esta colaboración, las fundaciones deberían mejorar su sistema para proporcionar la financiación a las empresas sociales y profesionalizarse como los fondos de inversión.

El análisis de este caso ha revelado que, a lo largo de su existencia, LCDC evitaba financiarse mediante instrumentos de deuda. En concreto, la empresa no acudió a la financiación bancaria, ni de la banca convencional ni de la banca ética, ya que consideraba que era demasiado arriesgada y rígida. Los fundadores prefirieron acudir a los ahorros personales en las primeras etapas del ciclo de vida de LCDC para mantener su autonomía en la toma de decisiones y en la estrategia, a colaborar con la banca. En cuanto a la fase de crecimiento, acudieron a la financiación de los inversores de impacto a través de capital social, lo cual confirma que evitaron la deuda. Esta evidencia podría ser relevante únicamente para este caso, o evidenciar una tendencia que caracteriza a las empresas de la economía social en general.

\section{Conclusiones}

Las inversiones de impacto representan una nueva fórmula de financiación para proyectos que tengan un impacto social y/o medioambiental, además de una rentabilidad económica. Los inversores que invierten en este tipo de proyectos acostumbran a priorizar el impacto social o medioambiental a la rentabilidad económica. Es decir, están dispuestos a obtener una rentabilidad inferior a la que podrían obtener en una inversión convencional, siempre y cuando puedan compensarlo con la consecución de un impacto social o medioambiental medible.

El mercado de las inversiones de impacto, como cualquier otro mercado, es una combinación de demanda de capital para financiar proyectos generadores de impacto, de oferta de capital de impacto y de intermediarios que ayudan a conectar la oferta y la demanda. El mercado de las inversiones de impacto se ha desarrollado de una forma importante en los Estados Unidos y en el Reino Unido. Estos países han sido pioneros en la utilización de esta fórmula de financiación para proyectos sociales. 
Hemos realizado un análisis de la aplicación de las inversiones de impacto para financiar el crecimiento del estudio de diseño y publicidad, La Casa de Carlota. Durante sus cinco años de vida, esta empresa ha pasado de una start-up recién nacida, a una empresa con una amplia base de clientes y con una filial en Latinoamérica. LCDC ha llegado al punto de equilibrio en 2017, y ahora está en plena fase de expansión. Durante estos años, la estrategia de búsqueda de fuentes de financiación ha ido cambiando según las necesidades de la empresa. Y aunque hay ciertas similitudes con una start-up convencional, la estrategia de financiación siempre ha estado marcada por su finalidad de creación del impacto social. El análisis realizado ha puesto de manifiesto las dificultades de LCDC para poder medir el impacto social generado. Según sus fundadores, el impacto que genera la inserción en el mercado laboral de personas con discapacidad intelectual es evidente, pero no disponen de herramientas que permitan cuantificar este impacto. En este sentido, consideran que esta falta de recursos para la medición del impacto social puede ser un escollo importante para que las empresas sociales reciban financiación de los inversores de impacto.

LCDC se decidió por los inversores de impacto, en vez de la banca tradicional o el capital riesgo por la alineación de los objetivos, en el sentido de que tanto LCDC como sus inversores no buscaban la rentabilidad financiera a corto plazo, sino que priorizaban la creación del impacto social y la sostenibilidad económica a largo plazo. En el caso de LCDC, los inversores de impacto fueron dos fundaciones sin fines lucrativos, cuyas estrategias de inversión se caracterizan por ser conservadoras y cautelosas. Este hecho puede tener un impacto negativo en las empresas receptoras de sus inversiones, porque no suelen realizar grandes inyecciones de capital de una sola vez, sino que las distribuyen a lo largo de un periodo de tiempo. Este hecho afectó la estrategia de crecimiento de LCDC. Otra singularidad de la inversión de impacto fue la relación entre el inversor y la empresa social. En este sentido, LCDC trataba a sus inversores no solamente como proveedores de financiación, sino como socios, vinculando a los inversores en la actividad principal de la empresa, aprovechando su experiencia, y tratándolos como miembros del equipo.

Por último, la actuación de la plataforma intermediaria entre LCDC y las fundaciones inversoras resultó determinante para que la empresa pudiera financiarse a través de los inversores de impacto. En el caso de LCDC, fue la plataforma Ship2B, la que no solo facilitó la conexión de los dos agentes, sino que también ayudó en el desarrollo del plan de acción, la estimación del valor de la empresa y la aportación de los conocimientos técnicos al proyecto.

El estudio de caso analizado en este artículo ayuda a entender la naturaleza de las inversiones de impacto y arroja luz sobre este nuevo ecosistema. Las empresas sociales y los inversores de impacto ocupan un sitio importante en las economías de los países, tanto desarrollados como en desarrollo, y los investigadores académicos tienen que prestar más atención a este nuevo tipo de economía. 


\section{Bibliografía}

ALLMAN, K.A. \& ESCOBAR DE NOGALES, X. (2015): Impact Investment: a practical guide to investment process and social impact analysis, Hoboken, New Jersey: John Wiley \& Sons, Inc.

BASTIDA, R. (2018): Les inversions d'impacte (impact investments): Anàlsi de les inversions que generen valor econòmic, social i mediambiental, ACCID.

BELL, B. \& HAUGH, H. (2015): Exploring institutional field emergence: Insights from social investment, (Working Paper No. 1032), Oxford, UK.

BRANSDSTETTER, L. \& LEHNER, O.M. (2015): "Opening the market for impact invesments:the need for adapted portfolio tools", Entrepreneurship Research Journal, 5(2), 87-107.

CLARK, C., EMERSON, J. \& THORNLEY, B. (2015): The impact investor: lessons in leadership and strategy for collaborative capitalism, San Francisco, Jossey-Bass.

DAGGERS, J. \& NICHOLLS, A. (2016): "The landscape of social impact investment research: Trends and opportunities", SBS, Oxford.

DIOUF, D. (2015). "Exploring the Barriers to Impact Investing in the Sustainable Energy Area in West Africa." In: Decentralized Solutions for Developing Economies, Springer, 177-183.

EISENHARDT, K.M. (1989): "Building Theories from Case Study Research", Academy of Management Review, 14(4), 532-550. DOI: 10.5465/amr.1989.4308385.

EISENHARDT, K.M. \& GRAEBNER, M.E. (2007): "Theory Building From Cases: Opportunities And Challenges", Academy of Management Journal, 50(1), 25-32. DOI: 10.5465/amj.2007.24160888.

EPSTEIN, M.J. \& YUTHAS, K. (2017): Measuring and improving social impacts: a guide for nonprofits, companies and impact investors, London, Routledge, Taylor \& Francis Group. DOI: $10.4324 / 9781351276245$.

EUROSIF (2014): European SRI Study 2014, Eurosif, Brussels.

GIIN (2018): What is impact investing. Retrieved June 20, 2018, from url: https://thegiin.org/impactinvesting/need-to-know/\#what-is-impact-investing.

HÖCHSTÄDTER, A.K. \& SCHECK, B. (2015): "What's in a name: An analysis of impact investing understandings by academics and practitioners", Journal of Business Ethics, 132(2), 449-475. 
JACKSON, E.T. (2013): "Evaluating social impact bonds: questions, challenges, innovations, and possibilities in measuring outcomes in impact investing", Community Development, 44(5), 608616. DOI: $10.1080 / 15575330.2013 .854258$.

JOY, M. \& SHIELDS, J. (2013): "Social Impact Bonds: the next phase of third sector marketization", Canadian Journal of Nonprofit and Social Economy Research, 4(2), 39-55. DOI: $10.22230 / c j n s e r .2013 v 4 n 2 a 148$.

KRAMER, M.R. \& PORTER, M. (2011): "Creating shared value", Harvard business review, 89(1/2), 62-77.

LYONS, T.S. \& KICKUL, J.R. (2013): "The social enterprise financing landscape: The lay of the land and new research on the horizon", Entrepreneurship Research Journal, 3(2), 147-159.

MCHUGH, N., SINCLAIR, S., ROY, M., HUCKFIELD, L. \& DONALDSON, C. (2013): "Social impact bonds: a wolf in sheep's clothing?", Journal of Poverty and Social Justice, 21(3), 247-257.

McWADE, W. (2012): "The Role for Social Enterprises and Social Investors in the Development Struggle", Journal of Social Entrepreneurship, 3(1), 96-112. DOI: 10.1080/19420676.2012.663783.

MENDELL, M. \& BARBOSA, E. (2013): "Impact investing: a preliminary analysis of emergent primary and secondary exchange platforms", Journal of Sustainable Finance \& Investment, 3(2), 111-123. DOI: $10.1080 / 20430795.2013 .776258$.

MULGAN, G. (2015): "Social finance. Does 'investment' add value?", In: A. Nicholls, R. Paton, \& J. Emerson (Eds.), Social Finance, Oxford University Press. DOI: 10.1093/ acprof:0so/9780198703761.003.0002.

NICHOLLS, A. (2010): "The institutionalization of social investment: The interplay of investment logics and investor rationalities", Journal of Social Entrepreneurship, 1(1), 70-100. DOI: 10.1080/194206710037012571.

NICHOLLS, A. \& EMERSON, J. (2015a): "Impact Investing: a market in evolution". In: A. Nicholls, R. Paton, \& J. Emerson (Eds.), Social finance, Oxford University Press, 207-251.

NICHOLLS, A., \& EMERSON, J. (2015b): "Social finance: capitalizing social impact". In: A. Nicholls, R. Paton \& J. Emerson (Eds.), Social finance, Oxford University Press, 1-43. DOI: 10.1093/acprof: oso/9780198703761.003.0001.

NICHOLLS, A. \& SCHWARZ, R. (2014): "The demand side of the social investment marketplace". In: L.M. Salamon (Ed.), New frontiers of philanthropy: A guide to the new tools and new actors that are reshaping global philanthropy and social investing, New York: Oxford University Press, 562-582. DOI: 10.1093/acprof:oso/9780199357543.003.0021.

NICHOLLS, A., SPEAR, R. \& PATON, R. (2015): Public policy for social finance in context, DOI:10.1093/ acprof:0so/9780198703761.003.0015. 
OECD (2018): OECD Social Impact Investment Initiative, Organization for Economic Co-operation and Development. Accedido el dia 12 de diciembre de 2018, a https://www.oecd.org/dac/financingsustainable-development/development-finance-topics/social-impact-investment-initiative.htm.

ORMISTON, J. \& SEYMOUR, R.G. (2014): "The Emergence of Social Investment as a Moral System of Exchange: The Australian experience", Social Entrepreneurship and Enterprise: Concepts in Context, 301-323.

PATTON, M.Q. (2005): Qualitative research, Wiley Online Library. DOI: 10.1002/0470013192.bsa514

PRIEDE BERGAMINI, T., LÓPEZ-CÓZAR NAVARRO, C. \& RODRíGUEZ LÓPEZ, A. (2014): "Análisis del marco económico-jurídico específico para los emprendedores sociales. Un estudio comparado entre diversos países", CIRIEC-España, Revista de Economía Pública Social y Cooperativa, 80, 5-28.

RAJAN, A.T., KOSERWAL, P. \& KEERTHANA, S. (2014): "The Global epicenter of impact investing: An analysis of social venture investments in India", The Journal of Private Equity, 37-50.

SANCHEZ PACHÓN, L. \& PÉREZ CHINARRO, E. (2015): "Las entidades de economía social como protagonistas de un nuevo modelo de emprendimiento y medidas legales de apoyo al emprendimiento", CIRIEC-España, Revista de Economía Pública, Social y Cooperativa, 84, 3562.

SEDDON, F.A., HAZENBERG, R. \& DENNY, S. (2013): "What are the barriers to investing in social enterprises? An investigation into the attitudes and experiences of social entrepreneurs in the United Kingdom." In: 4th EMES European Research Network International Research Conference on Social Enterprise, University of Liege, Belgium, 01-04 July.

THE ECONOMIST (2017): "Sustainable investments joins the mainstream", November 25th.

VALCÁRCEL DUEÑAS, M. (2012): "Los fondos europeos de emprendimiento social: su aplicación en España", CIRIEC-España, Revista de Economía Pública, Social y Cooperativa, 75, 104-128.

YIN, R.K. (2017): Case study research and applications: Design and methods, Sage publications. 
